\title{
Discovery of SIPI6473, a New, Potent, and Orally Bioavailable Multikinase Inhibitor for the Treatment of Non-small Cell Lung Cancer
}

\author{
Xiu Gu ${ }^{1,2}$ Zi-Xue Zhang ${ }^{1} \quad$ Min-Ru Jiao ${ }^{1} \quad$ Xin-Yan Peng ${ }^{1} \quad$ Jian-Qi Li ${ }^{1} \quad$ Qing-Wei Zhang ${ }^{1 *}$
}

${ }^{1}$ Novel Technology Center of Pharmaceutical Chemistry, Shanghai Institute of Pharmaceutical Industry, China State Institute of Pharmaceutical Industry, Shanghai, People's Republic of China

2 School of Chemistry and Chemical Engineering, Shanghai University of Engineering Science, Shanghai, People's Republic of China
Address for correspondence Qing-Wei Zhang, PhD, Novel Technology Center of Pharmaceutical Chemistry, Shanghai Institute of Pharmaceutical Industry, 285 Gebaini Road, Shanghai 201203, People’s Republic of China (e-mail: sipiqingwei@163.com).

Pharmaceut Fronts 2021;3:e1-e7.

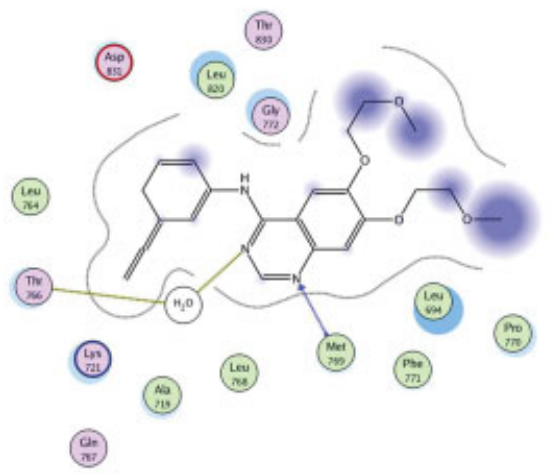

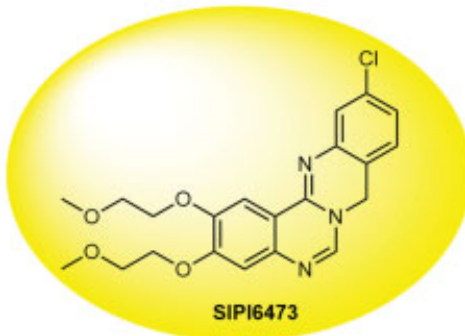

EGFR: IC $C_{50}=0.23 \mathrm{nmol} / \mathrm{L}$ FLT3: IC ${ }_{50}=230 \mathrm{nmol} / \mathrm{L}$ VEGF2: IC ${ }_{50}=440 \mathrm{nmol} / \mathrm{L}$ PDGFRa: $\mathrm{IC}_{50}=200 \mathrm{nmol} / \mathrm{L}$ hERG: IC ${ }_{50}=10.45 \mu \mathrm{mol} / \mathrm{L}$ $M T D>1500 \mathrm{mg} / \mathrm{kg}$

$T_{12}(p o)=2.32$ hours

$\mathrm{F}=\mathbf{2 1 . 0 8 \%}$

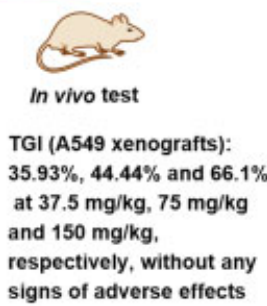

\section{Abstract \\ Keywords \\ - SIPI6473 \\ - multikinase inhibitor \\ - antitumor \\ - non-small cell lung cancer}

A novel series of quinazoline derivatives were designed, synthesized, and evaluated as multikinase inhibitors. Most of these compounds showed antiproliferation activities of several human cancer cell lines and exhibited inhibition efficacy against the estimated glomerular filtration rate (EGFR) in the nanomolar level. Among those compounds, compound B5 (also named SIPI6473) displayed the maximum effect, and thus was chosen for further study. Our data revealed that B5 inhibited the activity of several kinases (such as EGFR, VEGFR2, and PDGFR $\alpha$ ) that contributed to the development of non-small cell lung cancer (NSCLC). Besides, an in vivo study also showed that B5 inhibited tumor growth without signs of adverse effects in the A549 xenograft model. In conclusion, B5 may represent a new and promising drug for the treatment of NSCLC. received

March 9, 2021

accepted

May 8, 2021
DOI https://doi.org/ $10.1055 / \mathrm{s}-0041-1731081$ ISSN 2628-5088. (c) 2021. The Author(s).

This is an open access article published by Thieme under the terms of the Creative Commons Attribution License, permitting unrestricted use, distribution, and reproduction so long as the original work is properly cited. (https://creativecommons.org/licenses/by/4.0/)

Georg Thieme Verlag KG, Rüdigerstraße 14, 70469 Stuttgart,

Germany 


\section{Introduction}

Nitrogen-containing heterocycles, including quinazolinebased compounds, have gained great interest in targeted therapies as antitumor drugs. ${ }^{1-3}$ As a privileged scaffold, the 4-aminoquinazoline scaffold is an interesting pharmacophore, and widely used for making many specific kinase inhibitors, including tyrosine kinase, in medicinal chemistry for the treatment of lung, breast, colorectal cancers, etc. (-Fig. 1). ${ }^{4-6}$

Despite the clear clinical benefits, the efficacy of tyrosine kinase inhibitors (TKIs), such as Gefitinib or Erlotinib, in cancer treatment is eventually diminished because of acquired point mutations in the kinase domain of the estimated glomerular filtration rate (EGFR), and the subsequent induction of carcinogenic events, including ALK rearrangements, vascular EGFR (VEGFR) amplifications, platelet-derived growth factor receptor (PDGFR) amplifications, and so on. ${ }^{7-12}$ To our acknowledge, cancer has multiple pathologies with unique molecular signatures, thus, developing novel multitargeted TKIs targeting different signaling pathways and oncogenic drivers will be more efficient and beneficial than those obtained with single-target approaches. $^{13-16}$

It is unambiguously confirmed that the quinazoline core binds to a narrow hydrophobic pocket in the $\mathrm{N}$-terminal domain of EGFR protein, which is a common pharmacophoric feature of EGFR TKIs containing 4-aminoquinazoline core. The $\mathrm{N}-1$ and $\mathrm{N}-3$ nitrogen atoms of the quinazoline ring interact with amino acid residue $\mathrm{NH}$ or a bridging water through H-bonding, which played a crucial part in binding and the subsequent inhibitory capacity. Meanwhile, the 4-anilino group occupied a deep pocket at the back of the ATP-binding site and the C6 and/or C7-positions of quinazoline extended toward the entrance of the binding site of the protein. ${ }^{17-19}$

Up to now, most approaches have been adopted to enhance the potency and selectivity of these quinazoline derivatives in terms of differing functional groups on the C6 and $C 7$ regions or changing the substituents on the 4-anilino group. ${ }^{20-26}$ However, the exploration of the flexibility limitation of the $\mathrm{NH}$ spacer on 4-aminoquinazoline score is rarely reported. In fact, it would be essential to know how the flexibility of the $\mathrm{NH}$ spacer affects the TKI's inhibitory activity for further drug design.

In this article, we aim to develop a novel TKI skeleton according to the binding model of Erlotinib with EGFR protein. We designed and synthesized a novel series of quinazoline derivatives by means of conformational restriction for $\mathrm{NH}$ spacer and evaluated their antitumor activities (-Fig. 2).

\section{Results and Discussion}

The synthesis of series A and $\mathbf{B}$ target compounds is shown in Scheme 1. Coupling of chloride 1 with 4 -substituted indolines 2a-2d gave the desired compounds A1-A4. Chloride $\mathbf{1}$ was coupled with substituted 2-aminobenzyl alcohol 3a-3m to give the key intermediates $\mathbf{4 a - 4 m}$. Intramolecular cyclization of $\mathbf{4 a - 4 m}$ with phosphoryl chloride afforded the desired compounds B1-B13.

The effects of the target compounds on proliferation of different cancer cells were determined using MTT assay according to a reported study. ${ }^{23}$ Taxol and Erlotinib served as control drugs. Cancer cells used in this assay included K562 (human chronic myelogenous leukemia cells), Jurkat (E6-1) (human T lymphocyte leukemia cells), PANC-1 (human pancreatic carcinoma cells), A549 (human non-small cell lung cancer cells), Colo320 (human colorectal adenocarcinoma cells), Hut78 (human cutaneous lymphoma cells), MDA-MB-435s (human breast ductal carcinoma cells), Hep3B (human hepatoma cells), and PC-3 (human prostate cancer cells); and the concentration of the target compounds (A1-A4 and B1-B13) were $10 \mu \mathrm{mol} / \mathrm{L}$. As shown in -Table $\mathbf{1}$, most target compounds exhibited moderate to potent antiproliferative activities against the tested
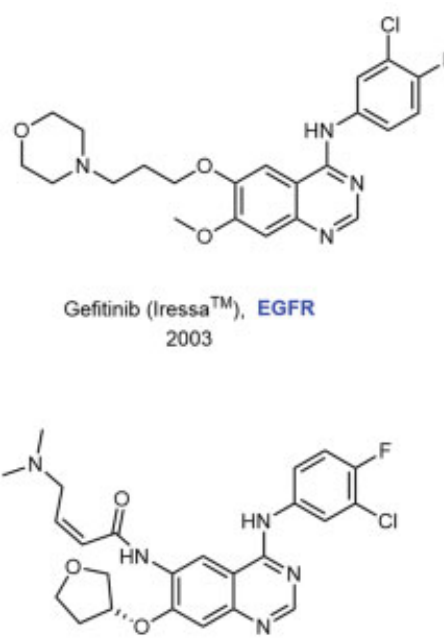

Afatinib (Gilotrif ${ }^{\mathrm{TM}}$ ), EGFR/HER2 2013
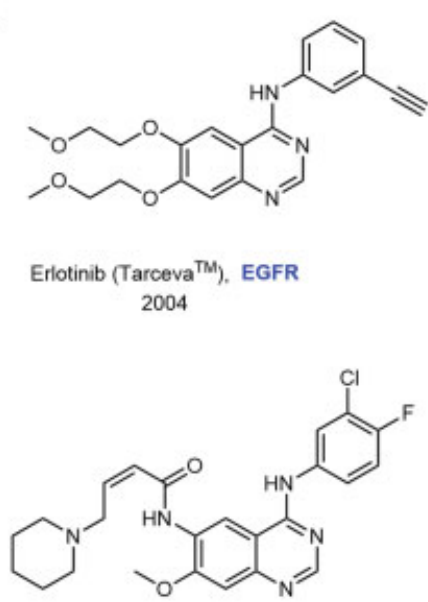

Dacomitinib (VizimproTM), EGFR/HER2/HER4 2018
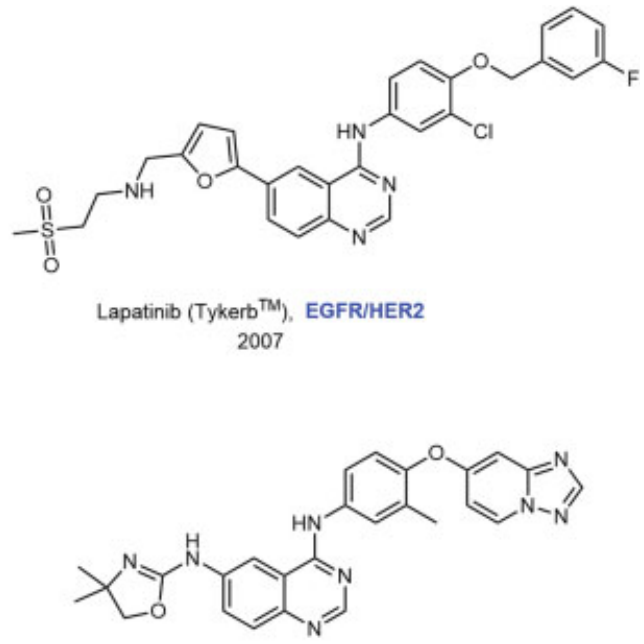

Tucatinib (Tukysa ${ }^{\text {TM}}$ ), HER2 2020

Fig. 1 Representative approved TKIs containing 4-aminoquinazoline group. TKIs, tyrosine kinase inhibitors. 


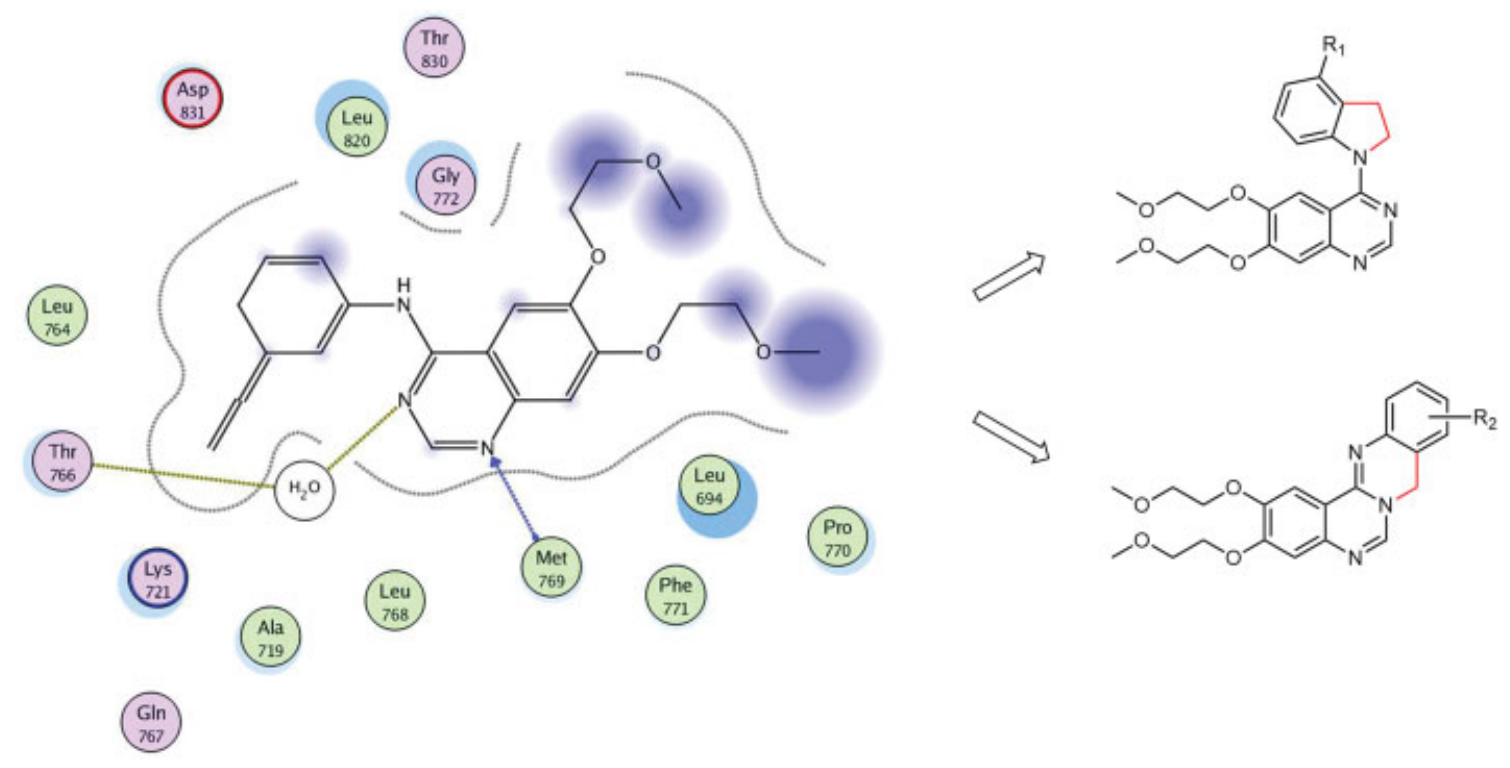

Fig. 2 According to the binding model of Erlotinib with EGFR protein, novel compounds were designed by conformational restriction. EGFR, estimated glomerular filtration rate.

cancer cells. At the same test concentration of $10 \mu \mathrm{mol} / \mathrm{L}$, most series B compounds significantly inhibited the cell proliferation against K562 cells, A549 cells, and MDA-MB-435s cells, and the potency of series $\mathbf{B}$ compounds was obviously superior to series A compounds and Erlotinib.

The $\mathrm{IC}_{50}$ values of the target compounds for EGFR activity were also determined. - Table 1 shows that incorporation of $\mathrm{N}$-containing indoline substituents to yield compounds A1-A4 resulted in the complete loss of EGFR inhibitory activities $\left(\mathrm{IC}_{50}>10 \mu \mathrm{mol} / \mathrm{L}\right)$. Interestingly, compounds B1-B13, only bearing a $\mathrm{N}-1$ nitrogen as $\mathrm{H}$-bond donor in the quinazoline fragment, maintained or slightly enhanced inhibitory activity against EGFR, indicating that N-3 nitrogen may not be critical in the common binding mode.
Besides, the preliminary structure-activity relationship of $\mathbf{B}$ series analogues with substituted groups at the phenyl nucleus was further investigated. Our data showed that the position of the substituents on the phenyl moiety had great effect on EGFR activities. For instance, the introduction of the same substituent at 11-position, in comparison with that at 9/10/12-position, could significantly improve the inhibitory activity against EGFR (compound B5 vs. B1, B3, B9, and B11). Meanwhile, a chlorine substituent on 11-position, in comparison with other substituents, showed stronger EGFR inhibition (compared B5 with B6, B7, and B8). Among them, compound B5 was the most potent compound ( $\mathrm{IC}_{50}$ value was $0.23 \mathrm{nmol} / \mathrm{L}$ ), in particular, which was over twofold more potent than Erlotinib $\left(\mathrm{IC}_{50}\right.$ value was $0.56 \mathrm{nmol} / \mathrm{L}$ ). Excitingly, compound $\mathbf{B 5}$ demonstrated the best

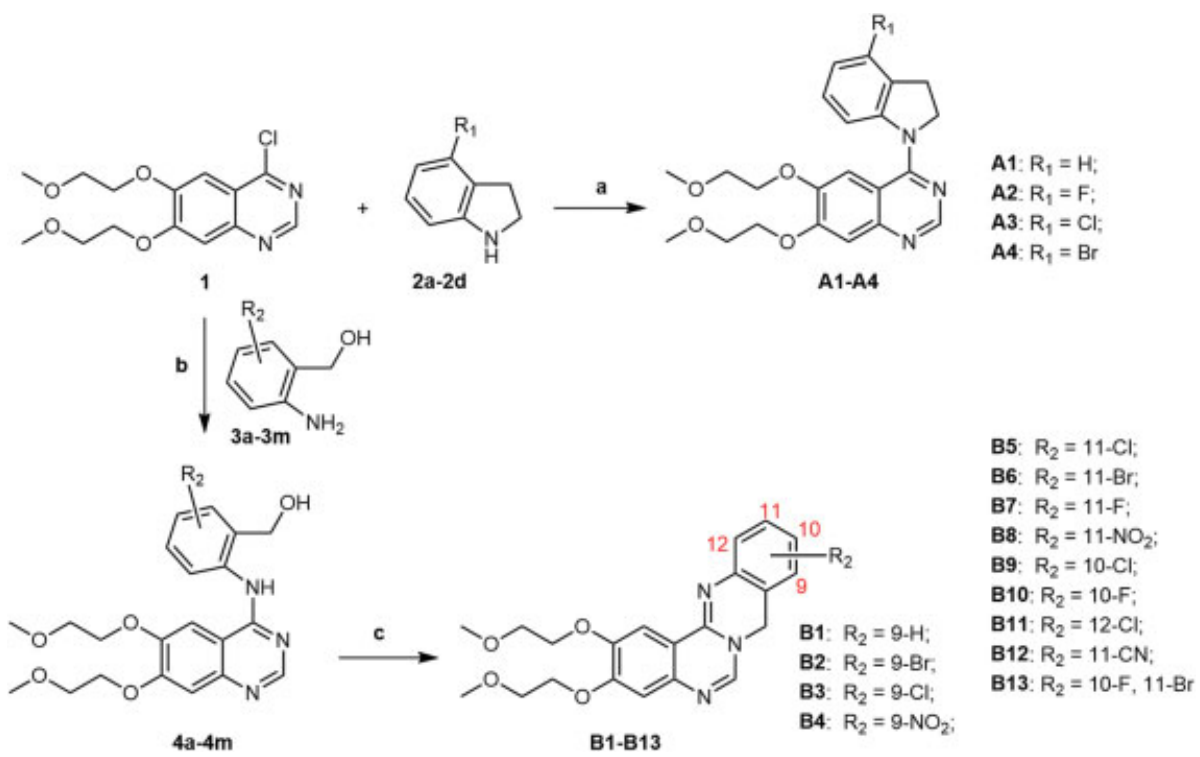

Scheme 1 Synthesis of compounds A1-A4 and B1-B13. Reagent and conditions: (a) DMF, $\mathrm{K}_{2} \mathrm{CO}_{3}, 130^{\circ} \mathrm{C}, 4$ hours; (b) isopropanol, reflux, 6 hours; (c) $\mathrm{POCl}_{3}$, reflux, 3 hours. 
Table 1 Antiproliferative activity and EGFR inhibitory activity of compounds A1-A4 and B1-B13 in different cell lines

\begin{tabular}{|c|c|c|c|c|c|c|c|c|c|c|}
\hline \multirow[t]{2}{*}{ Compound } & \multicolumn{9}{|c|}{ Inhibition ratio toward tumor cells/\% $(10 \mu \mathrm{mol} / \mathrm{L})^{a}$} & \multirow{2}{*}{$\begin{array}{l}\text { EGFR inhibitory } \\
\text { activity } \mathrm{IC}_{50} \\
(\mathrm{nmol} / \mathrm{L})^{\mathrm{b}}\end{array}$} \\
\hline & K562 & $\begin{array}{l}\text { Jurkat } \\
\text { (E6-1) }\end{array}$ & PANC-1 & A549 & Colo320 & Hut78 & MDA-MB-435s & Нер3В & PC-3 & \\
\hline A1 & 26.92 & 13.50 & -12.67 & 5.28 & 31.75 & 10.33 & 6.97 & -1.82 & 7.61 & $>10,000$ \\
\hline$A 2$ & 19.15 & 33.69 & 19.56 & 49.89 & 9.66 & -5.91 & 37.48 & -8.35 & 28.12 & $>10,000$ \\
\hline A3 & 13.63 & 26.63 & 22.38 & 20.69 & 52.71 & 44.27 & 48.94 & 20.33 & 33.50 & $>10,000$ \\
\hline A4 & 20.51 & 38.49 & -10.14 & 41.81 & -9.27 & 2.24 & -11.94 & 29.58 & 28.98 & $>10,000$ \\
\hline B1 & 75.98 & 40.80 & 10.30 & 86.06 & -1.56 & 2.78 & 49.51 & 36.48 & 58.15 & 1,710 \\
\hline B2 & 85.92 & 24.45 & 10.71 & 89.24 & 4.23 & 13.78 & 57.62 & 17.90 & 30.17 & 1,080 \\
\hline B3 & 101.24 & 98.17 & 10.10 & 99.04 & -9.27 & 79.30 & 65.32 & 97.53 & 52.08 & 2,730 \\
\hline B4 & 93.75 & 87.30 & 22.38 & 90.22 & 12.71 & 59.57 & 66.20 & 74.56 & 48.89 & 1,440 \\
\hline B5 & 98.46 & 97.97 & 15.92 & 91.08 & -9.70 & 71.39 & 67.92 & 72.34 & 87.74 & 0.23 \\
\hline B6 & 59.03 & 84.05 & 20.01 & 88.56 & 8.13 & 65.15 & 72.26 & 67.44 & 76.16 & 79.3 \\
\hline B7 & 61.10 & 31.12 & 19.74 & 80.69 & -6.99 & -3.79 & 71.59 & 19.00 & 42.73 & 393.2 \\
\hline B8 & 67.03 & 33.67 & 15.67 & 91.43 & -5.14 & -4.54 & 51.20 & 29.26 & 32.37 & 835.1 \\
\hline B9 & 37.58 & 9.79 & 11.59 & 79.83 & -8.91 & -5.01 & 42.57 & 70.35 & 71.65 & 7,500 \\
\hline B10 & 55.20 & 79.56 & 19.56 & 87.14 & 9.66 & 45.28 & 95.49 & 85.22 & 91.67 & 2,013 \\
\hline B11 & 68.27 & 48.86 & 17.08 & 82.45 & -1.70 & -3.55 & 90.61 & 14.40 & 53.57 & 1,060 \\
\hline B12 & 51.51 & 85.81 & 11.77 & 90.76 & 8.29 & 83.20 & 52.03 & 57.36 & 81.32 & 872.9 \\
\hline B13 & 69.67 & 72.82 & 8.96 & 91.65 & -7.93 & -67.18 & 90.95 & 45.08 & 12.99 & 4,538 \\
\hline Taxol & 67.23 & 93.18 & 65.64 & 82.88 & 68.52 & 69.92 & 45.97 & 62.29 & 72.65 & NT \\
\hline Erlotinib & 28.61 & 80.17 & 27.92 & 78.79 & 13.30 & 56.51 & 44.66 & 41.21 & 40.78 & 0.56 \\
\hline
\end{tabular}

Abbreviation: EGFR, estimated glomerular filtration rate.

${ }^{a}$ Values are means of three experiments.

${ }^{\mathrm{b}} \mathrm{IC}_{50}$ values are reported as the average of at least two separate determinations.

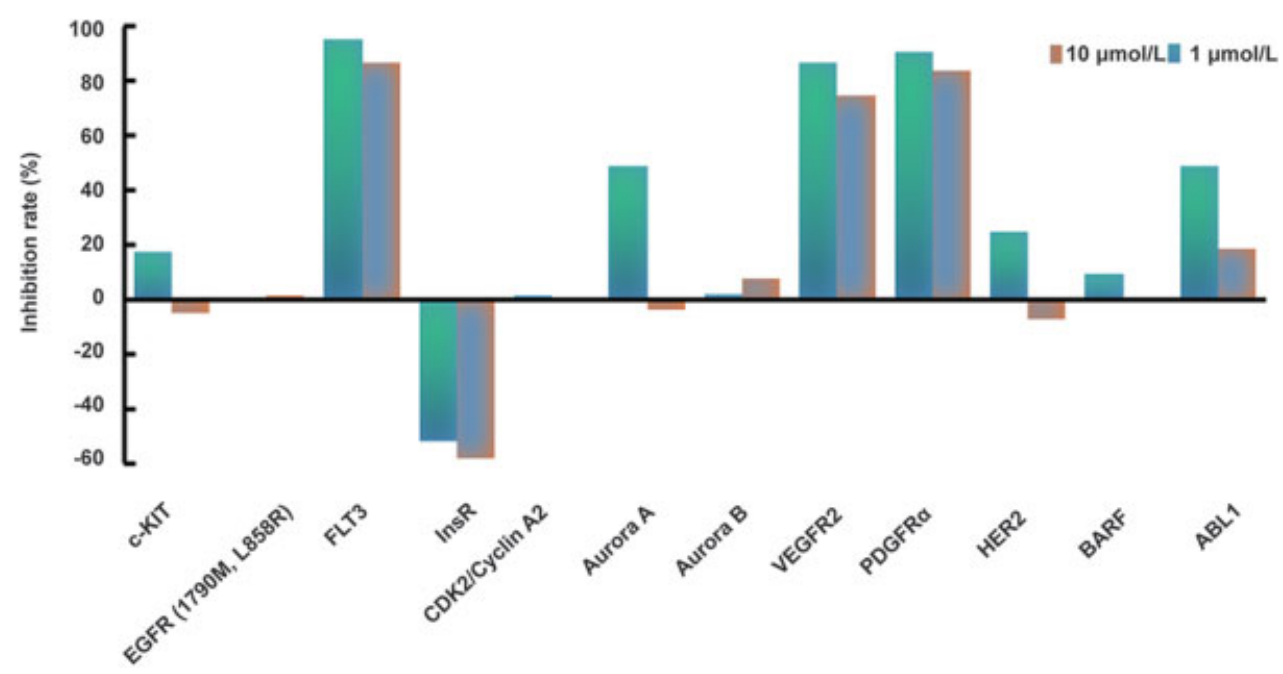

Fig. 3 Kinase assay results for compound B5.

inhibitory activity against cell assay for K562 cells, A549 cells, and MDA-MB-435s cells, and the effect of which was significantly superior to that of Erlotinib.

Accordingly, we were prompted to investigate whether this potency was specifically against EGFR. As shown in - Fig. 3, compound B5 strongly inhibited the kinase activity of FLT3, VEGFR2, and PDGFR $\alpha$ at the test concentration of $1 \mu \mathrm{mol} / \mathrm{L}$ (inhibition ratio $>50 \%$ ) with $\mathrm{IC}_{50}$ values being $0.23,0.44$, and $0.20 \mu \mathrm{mol} / \mathrm{L}$, respectively. Apparently, compound B5 was an anticancer multitargeted protein kinase inhibitor, which remarkably inhibits cell proliferation by targeting multiple kinases.

On the basis of the above results, we conducted acute toxicity test and hERG automated patch clamp (QPatch) assay 
Table 2 Profiles of compound B5 and Erlotinib

\begin{tabular}{|c|c|c|c|c|c|c|}
\hline \multirow[t]{2}{*}{ Compound } & \multirow{2}{*}{$\begin{array}{l}\text { MTD } \\
(\mathrm{mg} / \mathrm{kg})\end{array}$} & \multirow{2}{*}{$\begin{array}{l}\mathrm{IC}_{50}^{\mathrm{a}} \\
(\mu \mathrm{mol} / \mathrm{L}) \\
\mathrm{hERG}\end{array}$} & \multicolumn{4}{|c|}{ PK profiles ${ }^{b}$} \\
\hline & & & $\begin{array}{l}T_{1 / 2} \\
\text { po (h) }\end{array}$ & $\begin{array}{l}T_{\max } \\
\text { po (h) }\end{array}$ & $\begin{array}{l}C_{\max } \\
\text { po }(\mu g / L)\end{array}$ & $\begin{array}{l}F \\
(\%)\end{array}$ \\
\hline B5 & $>1,500$ & 10.46 & 2.32 & 0.44 & 743.93 & 21.08 \\
\hline Erlotinib & $-^{c}$ & 14.26 & 2.65 & 0.88 & 1478.06 & 66.60 \\
\hline
\end{tabular}

Abbreviations: MTD, maximum tolerance dose; PK, pharmacokinetics; po, oral.

${ }^{a}$ hERG patch clamp screen as described in Dubin et al. ${ }^{27} \mathrm{IC}_{50}$ values represent the concentration to inhibit $50 \%$ of hERG current (IKr). Numbers represent $\mathrm{IC}_{50}$ values generated from 3-point concentration response relationships in duplicate.

${ }^{\mathrm{b}}$ For pharmacokinetic study, blood was collected from rats at various time points up to 24 hours, and plasma samples were analyzed using an Agilent $1200 \mathrm{HPLC}$ system coupled with an Agilent 6410B triple quadruple mass spectrometer. A solution of $0.05 \mathrm{~N} \mathrm{HCl}$ in saline was used as the vehicle for both intravenous and oral dosing.

"The symbol "-" means that the MTD was not measured.

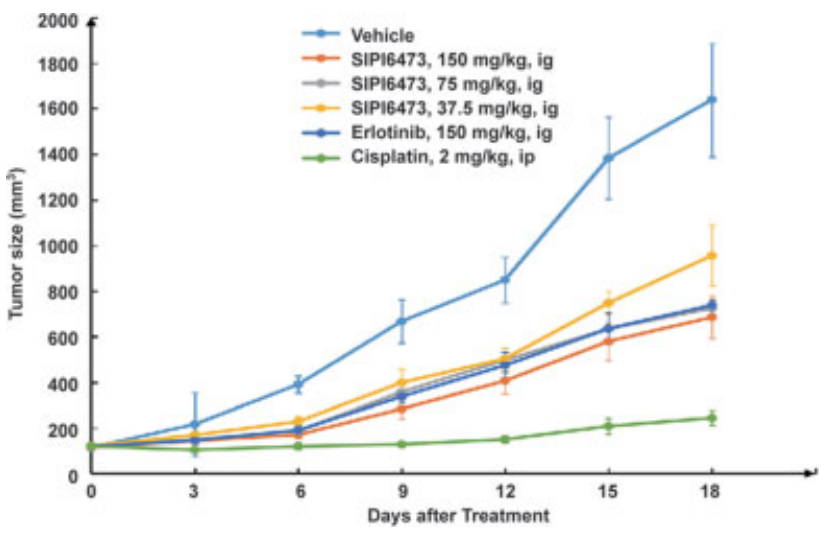

Fig. 4 Antitumor activity of B5 in mouse A549 xenograft model.

to examine the ancillary safety profiles of compound $\mathbf{B 5}$ as an anticancer agent. As is apparent from the data in - Table 2, compound $\mathbf{B 5}$ exhibited low toxicity to ICR mice with maximum tolerance dose value over $1,500 \mathrm{mg} / \mathrm{kg}$ by oral administration. Meanwhile, compound $\mathbf{B 5}$ displayed weak inhibitory activity against hERG $\left(\mathrm{IC}_{50}=10.46 \mu \mathrm{mol} / \mathrm{L}\right)$, similar to that of Erlotinib $\left(\mathrm{IC}_{50}=14.26 \mu \mathrm{mol} / \mathrm{L}\right)$.

The pharmacokinetic studies of compound $\mathbf{B 5}$ and Erlotinib were performed using female Sprague Dawley rats when dosed intravenously at both $1 \mathrm{mg} / \mathrm{kg}$ and orally at $20 \mathrm{mg} / \mathrm{kg}$ (-Table 2). The terminal phase half-life of $\mathbf{B 5}$ after po (oral) dosing was 2.32 hours. In contrast, po administration of Erlotinib led to a similar half-life $\left(t_{1 / 2}=2.65\right.$ hours). B5 was quickly absorbed after oral dosing, with a $T_{\max }$ of 0.44 hour. The oral bioavailability $(F)$ of $\mathbf{B 5}$ was $21.08 \%$, which is lower than that of Erlotinib ( $F=66.60 \%)$.

Given these promising pharmacokinetic parameters, the most promising compound B5 named SIPI6473 was further evaluated in the non-small cell lung cancer A549 xenograft mouse model at daily oral doses of $37.5,75$, and $150 \mathrm{mg} / \mathrm{kg}$ for 21 days (-Fig. 4). As shown in - Fig. 4, SIPI6473 could suppress tumor growth in a dose-dependent manner with tumor growth inhibition (TGI) ratios being 35.93, 44.44, and $66.1 \%$, respectively. Our data showed that the po administration of SIPI6473 at a high dose of $150 \mathrm{mg} / \mathrm{kg}$ resulted in a superior antitumor effect when compared with Erlotinib (TGI $=55.41 \%, 150 \mathrm{mg} / \mathrm{kg})$.

\section{Conclusion}

We designed and synthesized a series of novel quinazoline derivatives by conformational restriction. Among them, compound B5 (also named SIPI6473) showed strong proliferation activity in several cellular assays. SIPI6473 not only showed most potent EGFR inhibition activity but also exhibited nanomolar level inhibition against several other NSCLCrelated oncogene kinases, including VEGFR2 and PDGFR $\alpha$. In addition, in vivo studies showed that SIPI6473 had a good pharmacokinetic profile and displayed significant antitumor activity against A549 xenografts without signs of adverse effects. Hence, this new scaffold of potent protein kinase inhibitor SIPI6473 demonstrated a promising prospect for the discovery and development of new NSCLC agents and drugs. Further evaluation of SIPI6473 is ongoing and will be reported in due time.

\section{Experimental Section}

\section{General Synthetic Procedure of A1-A4}

The chloride 1 (1 mmol), indoline $(1.2 \mathrm{mmol})$, and $\mathrm{K}_{2} \mathrm{CO}_{3}$ ( $3 \mathrm{mmol}$ ) were dissolved in $15 \mathrm{~mL} \mathrm{DMF}$ and reacted at $130^{\circ} \mathrm{C}$ for 4 hours. After the reaction was complete, the reaction solution was poured into ice water, extracted with ethyl acetate $(50 \mathrm{~mL} \times 2)$, combined with organic phase, washed with water, saturated sodium carbonate solution, and saturated salt water $(10 \mathrm{~mL} \times 2)$, dried with anhydrous sodium sulfate, and concentrated, and by column chromatography (DCM: $\mathrm{MeOH}=50$ : 1-10: 1) a gray white solid A1 was obtained.

4-(Indolin-1-yl)-6,7-bis(2-methoxyethoxy)quinazoline (A1): ESI-MS $(m / z)$ : calcd. for $\mathrm{C}_{22} \mathrm{H}_{25} \mathrm{~N}_{3} \mathrm{O}_{4}[\mathrm{M}+\mathrm{H}]^{+}$; found 396.16. ${ }^{1} \mathrm{H}$ NMR (400 MHz, DMSO- $\left.d_{6}\right) \delta 8.59(\mathrm{~s}, 1 \mathrm{H}), 7.98$ (s, 1H), $7.60(\mathrm{~m}, 1 \mathrm{H}), 7.48(\mathrm{~s}, 1 \mathrm{H}), 7.38(\mathrm{~m}, 1 \mathrm{H}), 7.18-7.22$ $(\mathrm{m}, 2 \mathrm{H}), 4.60(\mathrm{~m}, 2 \mathrm{H}), 4.40(\mathrm{t}, J=4.4 \mathrm{~Hz}, 2 \mathrm{H}), 4.17$ $(\mathrm{t}, J=4.4 \mathrm{~Hz}, 2 \mathrm{H}), 3.85(\mathrm{t}, J=4.4 \mathrm{~Hz}, 2 \mathrm{H}), 3.78(\mathrm{t}, J=4.4 \mathrm{~Hz}$, $2 \mathrm{H}), 3.41-3.45(\mathrm{~s}, 6 \mathrm{H}), 3.31(\mathrm{t}, J=7.2 \mathrm{~Hz}, 2 \mathrm{H})$.

4-(4-Fluoroindolin-1-yl)-6,7-bis(2-methoxyethoxy)quinazoline (A2): ESI-MS $(m / z)$ : calcd. for $\mathrm{C}_{22} \mathrm{H}_{24} \mathrm{FN}_{3} \mathrm{O}_{4}[\mathrm{M}+$ $\mathrm{H}^{+}$; found 414.1661. ${ }^{1} \mathrm{H}$ NMR (400 MHz, DMSO- $\left.d_{6}\right) \delta 8.61$ $(\mathrm{s}, 1 \mathrm{H}), 8.05(\mathrm{~s}, 1 \mathrm{H}), 7.55(\mathrm{~s}, 1 \mathrm{H}), 7.45(\mathrm{~m}, 1 \mathrm{H}), 7.25(\mathrm{~m}, 1 \mathrm{H})$, $6.90(\mathrm{~m}, 1 \mathrm{H}), 4.66(\mathrm{t}, J=8.0 \mathrm{~Hz}, 2 \mathrm{H}), 4.45(\mathrm{t}, J=4.4 \mathrm{~Hz}, 2 \mathrm{H})$, 
$4.24(\mathrm{t}, J=4.8 \mathrm{~Hz}, 2 \mathrm{H}), 3.88(\mathrm{t}, J=4.4 \mathrm{~Hz}, 2 \mathrm{H}), 3.80(\mathrm{t}$, $J=4.4 \mathrm{~Hz}, 2 \mathrm{H}), 3.43-3.48(\mathrm{~s}, 6 \mathrm{H}), 3.30(\mathrm{t}, J=8.0 \mathrm{~Hz}, 2 \mathrm{H})$. 4-(4-Chloroindolin-1-yl)-6,7-bis(2-methoxyethoxy) quinazoline (A3): ESI-MS $(m / z)$ : calcd. for $\mathrm{C}_{22} \mathrm{H}_{24} \mathrm{ClN}_{3} \mathrm{O}_{4}$ $[\mathrm{M}+\mathrm{H}]^{+}$; found $430.1563 .{ }^{1} \mathrm{H}$ NMR $(400 \mathrm{MHz}$, DMSO-d 6 ) $\delta 8.65(\mathrm{~s}, 1 \mathrm{H}), 8.02(\mathrm{~s}, 1 \mathrm{H}), 7.55(\mathrm{t}, J=7.2 \mathrm{~Hz}, 1 \mathrm{H}), 7.45(\mathrm{~s}, 1 \mathrm{H})$, $7.25(\mathrm{~s}, 1 \mathrm{H}), 7.19-7.22(\mathrm{~m}, 1 \mathrm{H}), 4.66(\mathrm{t}, J=7.6 \mathrm{~Hz}, 2 \mathrm{H}), 4.46$ $(\mathrm{t}, J=4.4 \mathrm{~Hz}, 2 \mathrm{H}), 4.20(\mathrm{t}, J=4.4 \mathrm{~Hz}, 2 \mathrm{H}), 3.81(\mathrm{t}, J=4.4 \mathrm{~Hz}$, $2 \mathrm{H}), 3.47(\mathrm{~s}, 3 \mathrm{H}), 3.44(\mathrm{~s}, 3 \mathrm{H}), 3.34-3.38(\mathrm{t}, J=8.0 \mathrm{~Hz}, 2 \mathrm{H})$. 4-(4-Bromoindolin-1-yl)-6,7-bis(2-methoxyethoxy) quinazoline (A4): ESI-MS ( $m / z)$ : calcd. for $\mathrm{C}_{22} \mathrm{H}_{24} \mathrm{BrN}_{3} \mathrm{O}_{4}$ $[\mathrm{M}+\mathrm{H}]^{+}$; found 474.1077. ${ }^{1} \mathrm{H}$ NMR $\left(400 \mathrm{MHz}\right.$, DMSO- $\left.d_{6}\right) \boldsymbol{\delta}$ $8.52(\mathrm{~s}, 1 \mathrm{H}), 7.81(\mathrm{~s}, 1 \mathrm{H}), 7.60(\mathrm{t}, J=8.2 \mathrm{~Hz} 1 \mathrm{H}), 7.50(\mathrm{~s}, 1 \mathrm{H})$, $7.25(\mathrm{~m}, 1 \mathrm{H}), 7.05(\mathrm{t}, J=8.0 \mathrm{~Hz}, 1 \mathrm{H}), 4.64(\mathrm{t}, J=8.0 \mathrm{~Hz}, 2 \mathrm{H})$, $4.38(\mathrm{t}, J=4.4 \mathrm{~Hz}, 2 \mathrm{H}), 3.84(\mathrm{t}, 2 \mathrm{H}), 4.18(\mathrm{t}, 2 \mathrm{H}), 3.76(\mathrm{t}, 2 \mathrm{H})$, $3.42(\mathrm{~s}, 6 \mathrm{H}), 3.30(\mathrm{t}, J=7.6 \mathrm{~Hz}, 2 \mathrm{H})$.

\section{General Synthetic Procedure of B1-B13}

The chloride $\mathbf{1}$ (1.0 mmol) and 2-aminophenylethanol ( $1.0 \mathrm{mmol}$ ) were dissolved in $10 \mathrm{~mL}$ isopropanol, heated to reflux, and reacted for 6 hours. The reaction solution changed from turbid to clear and finally turbid. The white solid was obtained by recrystallization from ethyl acetate and then was dissolved in $5 \mathrm{~mL}$ of phosphorus oxychloride and heated to reflux for 3 hours. The heating was stopped, the unreacted phosphorus oxychloride evaporated, and the residue was added to ice water. $\mathrm{pH}$ was adjusted to 10 with saturated sodium carbonate solution. The mixture was extracted with ethyl acetate for 3 times, and the combined organic phase was washed with water, saturated salt water, and dried with anhydrous magnesium sulfate. After filtration, the filtrate was taken and concentrated under reduced pressure to obtain crude product. It was then recrystallized using ethanol to obtain B1 as a white solid with a yield of $50.47 \%$.

2,3-Bis(2-methoxyethoxy)-8H-quinazolino[4,3-b]quinazoline (B1): ESI-MS $(m / z)$ : calcd. for $\mathrm{C}_{22} \mathrm{H}_{23} \mathrm{~N}_{3} \mathrm{O}_{4}[\mathrm{M}+$ $\mathrm{H}]^{+}$; found 382.2090. ${ }^{1} \mathrm{H} \mathrm{NMR}\left(400 \mathrm{MHz}, \mathrm{DMSO}-d_{6}\right) \delta 9.16$ $(\mathrm{s}, 1 \mathrm{H}), 8.52(\mathrm{~s}, 1 \mathrm{H}), 7.17(\mathrm{~s}, 1 \mathrm{H}), 6.98-7.05(\mathrm{~m}, 2 \mathrm{H}), 6.86(\mathrm{~s}$, $1 \mathrm{H}), 4.70(\mathrm{~m}, 2 \mathrm{H}), 4.27-4.35(\mathrm{~m}, 4 \mathrm{H}), 3.83-3.93(\mathrm{~m}, 4 \mathrm{H})$, $3.46-3.48(\mathrm{~s}, 6 \mathrm{H})$.

9-Bromo-2,3-bis(2-methoxyethoxy)-8H-quinazolino [4,3-b]quinazoline (B2): ESI-MS $(\mathrm{m} / \mathrm{z})$ : calcd. for $\mathrm{C}_{21} \mathrm{H}_{22} \mathrm{BrN}_{3} \mathrm{O}_{4} \quad[\mathrm{M}+\mathrm{H}]^{+}$; found 460.086. ${ }^{1} \mathrm{H} \quad \mathrm{NMR}$ $\left(400 \mathrm{MHz}, \mathrm{DMSO}-d_{6}\right) \delta 8.02(\mathrm{~s}, 1 \mathrm{H}), 7.75(\mathrm{~s}, 1 \mathrm{H}), 7.30(\mathrm{~s}$, $1 \mathrm{H}), 7.20(\mathrm{~m}, 1 \mathrm{H}), 7.05-7.10(\mathrm{~m}, 2 \mathrm{H}), 5.55(\mathrm{~s}, 2 \mathrm{H}), 4.34(\mathrm{~m}$, $2 \mathrm{H}), 4.27(\mathrm{~m}, 2 \mathrm{H}), 3.83-3.90(\mathrm{~m}, 4 \mathrm{H}), 3.49(\mathrm{~s}, 6 \mathrm{H})$.

9-Chloro-2,3-bis(2-methoxyethoxy)-8H-quinazolino [4,3-b]quinazoline (B3): ESI-MS $(\mathrm{m} / \mathrm{z})$ : calcd. for $\mathrm{C}_{21} \mathrm{H}_{22} \mathrm{ClN}_{3} \mathrm{O}_{4} \quad[\mathrm{M}+\mathrm{H}]^{+}$; found 416.1401. ${ }^{1} \mathrm{H} \quad \mathrm{NMR}$ $\left(400 \mathrm{MHz}, \mathrm{DMSO}-d_{6}\right) \delta 8.05(\mathrm{~s}, 1 \mathrm{H}), 7.91(\mathrm{~s}, 1 \mathrm{H}), 7.37$ (s, $1 \mathrm{H}), 7.20(\mathrm{~m}, 1 \mathrm{H}), 7.07-7.10(\mathrm{~m}, 2 \mathrm{H}), 5.32(\mathrm{~s}, 2 \mathrm{H})$, $4.38-4.40(\mathrm{~m}, 2 \mathrm{H}), 4.27-4.31(\mathrm{~m}, 2 \mathrm{H}), 3.84-3.89(\mathrm{~m}$, $4 \mathrm{H}), 3.49(\mathrm{~s}, 6 \mathrm{H})$.

2,3-Bis(2-methoxyethoxy)-9-nitro-8H-quinazolino [4,3-b]quinazoline (B4): ESI-MS $(\mathrm{m} / \mathrm{z})$ : calcd. for $\mathrm{C}_{21} \mathrm{H}_{22} \mathrm{~N}_{4} \mathrm{O}_{6} \quad[\mathrm{M}+\mathrm{H}]^{+}$; found 427.1654. ${ }^{1} \mathrm{H} \quad \mathrm{NMR}$ $\left(400 \mathrm{MHz}, \mathrm{DMSO}-d_{6}\right) \delta 7.63(\mathrm{~s}, 1 \mathrm{H}), 7.55(\mathrm{~m}, 2 \mathrm{H}), 7.27$ (m, 1H), $7.15(\mathrm{~m}, 1 \mathrm{H}), 6.81(\mathrm{~s}, 1 \mathrm{H}), 5.42(\mathrm{~s}, 2 \mathrm{H}), 4.10(\mathrm{~m}$, $4 \mathrm{H}), 3.60(\mathrm{~m}, 4 \mathrm{H}), 3.25(\mathrm{~s}, 6 \mathrm{H})$.

11-Chloro-2,3-bis(2-methoxyethoxy)-8H-quinazolino [4,3-b]quinazoline (B5): ESI-MS $(\mathrm{m} / \mathrm{z})$ : calcd. for $\mathrm{C}_{21} \mathrm{H}_{22} \mathrm{ClN}_{3} \mathrm{O}_{4} \quad[\mathrm{M}+\mathrm{H}]^{+}$; found 416.1485. ${ }^{1} \mathrm{H} \quad \mathrm{NMR}$ $\left(400 \mathrm{MHz}, \mathrm{DMSO}-d_{6}\right) \delta 7.81(\mathrm{~s}, 1 \mathrm{H}), 7.60(\mathrm{~s}, 1 \mathrm{H}), 7.25(\mathrm{~s}$, $1 \mathrm{H}), 7.00(\mathrm{~m}, 2 \mathrm{H}), 6.90(\mathrm{~m}, 1 \mathrm{H}), 5.19(\mathrm{~s}, 2 \mathrm{H}), 4.25-4.30(\mathrm{~m}$, $4 \mathrm{H}), 3.85(\mathrm{~m}, 4 \mathrm{H}), 3.50(\mathrm{~s}, 6 \mathrm{H})$.

11-Bromo-2,3-bis(2-methoxyethoxy)-8H-quinazolino [4,3-b]quinazoline (B6): ESI-MS $(\mathrm{m} / \mathrm{z})$ : calcd. for $\mathrm{C}_{21} \mathrm{H}_{22} \mathrm{BrN}_{3} \mathrm{O}_{4} \quad[\mathrm{M}+\mathrm{H}]^{+}$; found 460.0213. ${ }^{1} \mathrm{H} \quad \mathrm{NMR}$ $\left(400 \mathrm{MHz}, \mathrm{DMSO}-d_{6}\right) \delta 8.88(\mathrm{~s}, 1 \mathrm{H}), 8.45(\mathrm{~s}, 1 \mathrm{H}), 8.41$ (s, $1 \mathrm{H}), 7.31(\mathrm{~m}, 2 \mathrm{H}), 7.13(\mathrm{~m}, J=8.0 \mathrm{~Hz}, 1 \mathrm{H}), 5.63(\mathrm{~s}, 2 \mathrm{H})$, 4.35-4.56 (m, 4H), $3.86(\mathrm{~m}, 4 \mathrm{H}), 3.46(\mathrm{~s}, 6 \mathrm{H})$.

11-Fluoro-2,3-bis(2-methoxyethoxy)-8H-quinazolino [4,3-b]quinazoline (B7): ESI-MS $(\mathrm{m} / \mathrm{z})$ : calcd. for $\mathrm{C}_{21} \mathrm{H}_{22} \mathrm{FN}_{3} \mathrm{O}_{4}[\mathrm{M}+\mathrm{H}]^{+}$; found 400.17. ${ }^{1} \mathrm{H}$ NMR (400 MHz, DMSO- $\left.d_{6}\right) \delta 8.59(\mathrm{~s}, 2 \mathrm{H}), 7.75(\mathrm{~s}, 1 \mathrm{H}), 7.49-7.38(\mathrm{~m}, 2 \mathrm{H}), 7.13$ (dd, $J=16.4,7.9 \mathrm{~Hz}, 1 \mathrm{H}), 5.57(\mathrm{~s}, 2 \mathrm{H}), 4.45-4.36(\mathrm{~m}, 4 \mathrm{H})$, 3.79-3.75 (m, $4 \mathrm{H}), 3.36(\mathrm{~s}, 3 \mathrm{H}), 3.35(\mathrm{~s}, 3 \mathrm{H})$.

2,3-Bis(2-methoxyethoxy)-11-nitro-8H-quinazolino [4,3-b]quinazoline (B8): ESI-MS $(\mathrm{m} / \mathrm{z})$ : calcd. for $\mathrm{C}_{21} \mathrm{H}_{22} \mathrm{~N}_{4} \mathrm{O}_{6}[\mathrm{M}+\mathrm{H}]^{+}$; found 427.1708. ${ }^{1} \mathrm{H} \mathrm{NMR}(600 \mathrm{MHz}$, $\left.\mathrm{CDCl}_{3}\right) \delta 8.05(\mathrm{~s}, 1 \mathrm{H}), 7.90(\mathrm{~s}, 1 \mathrm{H}), 7.78(\mathrm{q}, J=8.9,7.3 \mathrm{~Hz}, 2 \mathrm{H})$, $7.18(\mathrm{~d}, J=8.2 \mathrm{~Hz}, 1 \mathrm{H}), 6.97(\mathrm{~s}, 1 \mathrm{H}), 5.37-5.30(\mathrm{~m}, 2 \mathrm{H}), 4.17$ (q, $J=4.5 \mathrm{~Hz}, 2 \mathrm{H}), 4.11$ (dd, $J=6.0,3.4 \mathrm{~Hz}, 2 \mathrm{H}), 3.67$ (td, $J=5.6,2.8 \mathrm{~Hz}, 4 \mathrm{H}), 3.29-3.27(\mathrm{~m}, 6 \mathrm{H})$.

10-Chloro-2,3-bis(2-methoxyethoxy)-8H-quinazolino [4,3-b]quinazoline (B9): ESI-MS $(\mathrm{m} / \mathrm{z})$ : calcd. for $\mathrm{C}_{21} \mathrm{H}_{22} \mathrm{ClN}_{3} \mathrm{O}_{4} \quad[\mathrm{M}+\mathrm{H}]^{+} ;$found 416.16. ${ }^{1} \mathrm{H} \quad \mathrm{NMR}$ $\left(400 \mathrm{MHz}, \mathrm{DMSO}-d_{6}\right) \delta 7.86(\mathrm{~s}, 1 \mathrm{H}), 7.61(\mathrm{~s}, 1 \mathrm{H}), 7.23(\mathrm{~s}$, $1 \mathrm{H}), 7.04(\mathrm{~m}, 2 \mathrm{H}), 6.93(\mathrm{~m}, 1 \mathrm{H}), 5.21(\mathrm{~s}, 2 \mathrm{H}), 4.25-4.31(\mathrm{~m}$, $4 \mathrm{H}), 3.85(\mathrm{~m}, 4 \mathrm{H}), 3.48(\mathrm{~s}, 6 \mathrm{H})$.

10-Fluoro-2,3-bis(2-methoxyethoxy)-8H-quinazolino [4,3-b]quinazoline (B10): ESI-MS $(\mathrm{m} / \mathrm{z})$ : calcd. for $\mathrm{C}_{21} \mathrm{H}_{22} \mathrm{FN}_{3} \mathrm{O}_{4} \quad[\mathrm{M}+\mathrm{H}]^{+}$; found 400.1204. ${ }^{1} \mathrm{H} \quad \mathrm{NMR}$ $\left(400 \mathrm{MHz}, \mathrm{DMSO}-d_{6}\right) \delta 8.53(\mathrm{~s}, 1 \mathrm{H}), 7.75(\mathrm{~s}, 1 \mathrm{H}), 7.66(\mathrm{~s}$, $2 \mathrm{H}), 7.49-7.38(\mathrm{~m}, 1 \mathrm{H}), 7.13(\mathrm{~m}, 1 \mathrm{H}), 5.51(\mathrm{~s}, 2 \mathrm{H})$, 4.47-4.39 (m, 4H), $3.78(\mathrm{~m}, 4 \mathrm{H}), 3.41(\mathrm{~s}, 3 \mathrm{H}), 3.37(\mathrm{~s}, 3 \mathrm{H})$. 12-Chloro-2,3-bis(2-methoxyethoxy)-8H-quinazolino [4,3-b]quinazoline (B11): ESI-MS $(\mathrm{m} / \mathrm{z})$ : calcd. for $\mathrm{C}_{21} \mathrm{H}_{22} \mathrm{ClN}_{3} \mathrm{O}_{4} \quad[\mathrm{M}+\mathrm{H}]^{+}$; found 416.1469. ${ }^{1} \mathrm{H} \quad \mathrm{NMR}$ $\left(600 \mathrm{MHz}, \mathrm{CDCl}_{3}\right) \delta 9.33(\mathrm{~d}, J=9.0 \mathrm{~Hz}, 1 \mathrm{H}), 8.27(\mathrm{dt}, J=8.1$, $2.2 \mathrm{~Hz}, 1 \mathrm{H}), 8.17$ (s, 1H), 7.91-7.89 (m, 1H), 7.37-7.34 (m, $1 \mathrm{H}), 7.25-7.23(\mathrm{~m}, 1 \mathrm{H}), 4.39(\mathrm{~d}, J=4.6 \mathrm{~Hz}, 2 \mathrm{H}), 4.32(\mathrm{~d}$, $J=4.6 \mathrm{~Hz}, 2 \mathrm{H}), 3.92(\mathrm{t}, J=4.7 \mathrm{~Hz}, 2 \mathrm{H}), 3.90-3.89(\mathrm{~m}, 2 \mathrm{H})$, 3.85-3.78 (m, 2H), $3.54(\mathrm{~s}, 3 \mathrm{H}), 3.52(\mathrm{~s}, 3 \mathrm{H})$.

2,3-Bis(2-methoxyethoxy)-8H-quinazolino[4,3-b]quinazoline-11-carbonitrile (B12): ESI-MS $(\mathrm{m} / \mathrm{z})$ : calcd. for $\mathrm{C}_{22} \mathrm{H}_{22} \mathrm{ClN}_{4} \mathrm{O}_{4} \quad[\mathrm{M}+\mathrm{H}]^{+}$; found 407.1738. ${ }^{1} \mathrm{H} \quad \mathrm{NMR}$ $\left(600 \mathrm{MHz}, \mathrm{CDCl}_{3}\right) \delta 7.74(\mathrm{~s}, 1 \mathrm{H}), 7.59(\mathrm{~s}, 1 \mathrm{H}), 7.46(\mathrm{~d}, J=1.6$ $\mathrm{Hz}, 1 \mathrm{H}), 7.30-7.26$ (m, 1H), 7.07 (d, J=7.7 Hz, 1H), 6.97 (s, $1 \mathrm{H}), 5.23(\mathrm{~s}, 2 \mathrm{H}), 4.31(\mathrm{t}, J=4.7 \mathrm{~Hz}, 2 \mathrm{H}), 4.22(\mathrm{t}, J=4.7 \mathrm{~Hz}$, $2 \mathrm{H}), 3.84(\mathrm{q}, J=4.8 \mathrm{~Hz}, 4 \mathrm{H}), 3.50(\mathrm{~s}, 3 \mathrm{H}), 3.49(\mathrm{~s}, 3 \mathrm{H})$. 11-Bromo-10-fluoro-2,3-bis(2-methoxyethoxy)-8Hquinazolino[4,3-b]quinazoline (B13): ESI-MS ( $\mathrm{m} / \mathrm{z})$ : calcd. for $\mathrm{C}_{22} \mathrm{H}_{22} \mathrm{ClN}_{4} \mathrm{O}_{4}[\mathrm{M}+\mathrm{H}]^{+}$; found 478.00 . ${ }^{1} \mathrm{H}$ 
$\operatorname{NMR}\left(600 \mathrm{MHz}, \mathrm{CDCl}_{3}+\mathrm{CD}_{3} \mathrm{OD} \mathrm{LOCKCDCl}\right)_{3} \boldsymbol{\delta} 7.92(\mathrm{~s}, 2 \mathrm{H})$, $6.93(\mathrm{~s}, 2 \mathrm{H}), 5.27(\mathrm{~s}, 2 \mathrm{H}), 4.10(\mathrm{~m}, 4 \mathrm{H}), 3.64-3.60(\mathrm{~m}, 4 \mathrm{H})$, $3.25(\mathrm{~s}, 3 \mathrm{H}), 3.23(\mathrm{~s}, 3 \mathrm{H})$.

Funding

This work was financially supported by the National Science and Technology Major Project (Grant No. 2018ZX09711002-002-009), the National Natural Science Foundation of China (Grant No. 81703358), and Science and Technology Commission of Shanghai Municipality (Grant No. 17431903900, 18QB1404200,18ZR1437700).

\section{Conflict of Interest}

None.

\section{References}

1 Shagufta AI, Ahmad I. An insight into the therapeutic potential of quinazoline derivatives as anticancer agents. Med Chem Comm 2017;8(05):871-885

2 Ravez S, Castillo-Aguilera O, Depreux P, Goossens L. Quinazoline derivatives as anticancer drugs: a patent review (2011 - present). Expert Opin Ther Pat 2015;25(07):789-804

3 Marzaro G, Guiotto A, Chilin A. Quinazoline derivatives as potential anticancer agents: a patent review (2007 - 2010). Expert Opin Ther Pat 2012;22(03):223-252

4 Das D, Hong J. Recent advancements of 4-aminoquinazoline derivatives as kinase inhibitors and their applications in medicinal chemistry. Eur J Med Chem 2019;170:55-72

5 Chandregowda V, Kush AK, Chandrasekara Reddy G. Synthesis and in vitro antitumor activities of novel 4-anilinoquinazoline derivatives. Eur J Med Chem 2009;44(07):3046-3055

6 Ding C, Li D, Wang YW, et al. Discovery of ErbB/HDAC inhibitors by combining the core pharmacophores of HDAC inhibitor vorinostat and kinase inhibitors vandetanib, BMS-690514, neratinib, and TAK-285. Chin Chem Lett 2017;28(06):1220-1227

7 Kobayashi S, Boggon TJ, Dayaram T, et al. EGFR mutation and resistance of non-small-cell lung cancer to gefitinib. N Engl J Med 2005;352(08):786-792

8 Gainor JF, Varghese AM, Ou SH, et al. ALK rearrangements are mutually exclusive with mutations in EGFR or KRAS: an analysis of 1,683 patients with non-small cell lung cancer. Clin Cancer Res 2013;19(15):4273-4281

9 Naumov GN, Nilsson MB, Cascone T, et al. Combined vascular endothelial growth factor receptor and epidermal growth factor receptor (EGFR) blockade inhibits tumor growth in xenograft models of EGFR inhibitor resistance. Clin Cancer Res 2009;15(10):3484-3494

10 Akhavan D, Pourzia AL, Nourian AA, et al. De-repression of PDGFR $\beta$ transcription promotes acquired resistance to EGFR tyrosine kinase inhibitors in glioblastoma patients. Cancer Discov 2013;3(05):534-547

11 Ware KE, Hinz TK, Kleczko E, et al. A mechanism of resistance to gefitinib mediated by cellular reprogramming and the acquisition of an FGF2-FGFR1 autocrine growth loop. Oncogenesis 2013;2(03):e39

12 Bean J, Brennan C, Shih JY, et al. MET amplification occurs with or without T790M mutations in EGFR mutant lung tumors with acquired resistance to gefitinib or erlotinib. Proc Natl Acad Sci U S A 2007;104(52):20932-20937

13 Gentile C, Martorana A, Lauria A, Bonsignore R. Kinase inhibitors in multitargeted cancer therapy. Curr Med Chem 2017;24(16): 1671-1686

14 Medinger M, Drevs J. Receptor tyrosine kinases and anticancer therapy. Curr Pharm Des 2005;11(09):1139-1149

15 Markowitz JN, Fancher KM. Cabozantinib: a multitargeted oral tyrosine kinase inhibitor. Pharmacotherapy 2018;38(03):357-369

16 Skouras VS, Maragkos C, Grapsa D, Syrigos KN. Targeting neovasculature with multitargeted antiangiogenesis tyrosine kinase inhibitors in non-small cell lung cancer. BioDrugs 2016;30(05): 421-439

17 Sun HP, Zhu J, Chen YD, et al. Docking study and three-dimensional quantitative structure-activity relationship (3D-QSAR) analyses and novel molecular design of a series of 4-aminoquinazolines as inhibitors of Aurora B kinase. Chin J Chem 2011;29(09):1785-1799

18 Reddy PS, Lokhande KB, Nagar S, Reddy VD, Murthy PS, Swamy KV. Molecular modeling, docking, dynamics and simulation of gefitinib and its derivatives with EGFR in non-small cell lung cancer. Curr Comput Aided Drug Des 2018;14(03):246-252

19 Dömötör O, Pelivan K, Borics A, Keppler BK, Kowol CR, Enyedy ÉA Comparative studies on the human serum albumin binding of the clinically approved EGFR inhibitors gefitinib, erlotinib, afatinib, osimertinib and the investigational inhibitor KP2187. J Pharm Biomed Anal 2018;154:321-331

20 Smaill JB, Gonzales AJ, Spicer JA, et al. Tyrosine kinase inhibitors. 20. Optimization of substituted quinazoline and pyrido[3,4-d] pyrimidine derivatives as orally active, irreversible inhibitors of the epidermal growth factor receptor family. J Med Chem 2016;59 (17):8103-8124

21 Smaill JB, Palmer BD, Rewcastle GW, et al. Tyrosine kinase inhibitors. 15. 4-(Phenylamino)quinazoline and 4-(phenylamino)pyrido[d]pyrimidine acrylamides as irreversible inhibitors of the ATP binding site of the epidermal growth factor receptor. J Med Chem 1999;42(10):1803-1815

22 Chilin A, Conconi MT, Marzaro G, et al. Exploring epidermal growth factor receptor (EGFR) inhibitor features: the role of fused dioxygenated rings on the quinazoline scaffold. J Med Chem 2010; 53(04):1862-1866

23 Chang J, Ren H, Zhao M, et al. Development of a series of novel 4anlinoquinazoline derivatives possessing quinazoline skeleton: design, synthesis, EGFR kinase inhibitory efficacy, and evaluation of anticancer activities in vitro. Eur J Med Chem 2017;138:669-688

24 Li DD, Fang F, Li JR, et al. Discovery of 6-substituted 4-anilinoquinazolines with dioxygenated rings as novel EGFR tyrosine kinase inhibitors. Bioorg Med Chem Lett 2012;22(18):5870-5875

25 Zhang HQ, Gong FH, Li CG, et al. Design and discovery of 4anilinoquinazoline-acylamino derivatives as EGFR and VEGFR-2 dual TK inhibitors. Eur J Med Chem 2016;109:371-379

26 Abouzid K, Shouman S. Design, synthesis and in vitro antitumor activity of 4-aminoquinoline and 4-aminoquinazoline derivatives targeting EGFR tyrosine kinase. Bioorg Med Chem 2008;16(16): 7543-7551

27 Dubin AE, Nasser N, Rohrbacher J, et al. Identifying modulators of hERG channel activity using the PatchXpress planar patch clamp. J Biomol Screen 2005;10(02):168-181 